\title{
Recent Advances in Percutaneous Cardioscopy
}

\author{
Yasumi Uchida
}

Published online: 12 May 2011

(C) The Author(s) 2011. This article is published with open access at Springerlink.com

\begin{abstract}
Percutaneous cardioscopy, using high-resolution fiberoptic imaging, enables direct visualization of the cardiac interior, thereby enabling macroscopic pathological diagnosis. Percutaneous cardioscopy has demonstrated that the endocardial surface exhibits various colors characteristic of different heart diseases. This imaging modality can now be used for evaluation of the severity of myocardial ischemia, and staging of myocarditis. Myocardial blood flow recovery induced by vasodilating agents or percutaneous coronary interventions can be clearly visualized. Morphological and functional changes in the cardiac valves can also be evaluated. Cardioscope-guided endomyocardial biopsy enables pin-point biopsy of the diseased myocardium. Recently, dye-image cardioscopy and fluorescence cardioscopy were developed for evaluation of the subendocardial microcirculation. Cardioscope-guided intracardiac therapies such as myotomy, myectomy, valvulotomy, and transendocardial angiogenic and myogenic therapy have been trialed using animal models in anticipation of future clinical applications. Percutaneous cardioscopy has the potential to contribute to our understanding of heart disease, and to assist in guidance for intracardiac therapies.
\end{abstract}

Keywords Percutaneous cardioscopy $\cdot$ Dye-staining cardioscopy · Fluorescence cardioscopy · Cardioscopeguided endomyocardial biopsy and intracardiac surgery

\section{Introduction}

Direct observation of changes in the beating heart was previously beyond the scope of any available imaging

\section{Y. Uchida $(\triangle)$}

Japan Foundation for Cardiovascular Research,

2-30-17, Narashinodai,

Funabashi 274-0063, Japan

e-mail: uchiy@ta2.so-net.ne.jp modalities. Percutaneous cardioscopy, using high-resolution fiberoptic imaging, enables direct visualization of the cardiac interior, thereby enabling macroscopic pathological diagnosis. This imaging modality is now clinically employed not only for diagnosis of myocardial and valvular disease, but also for evaluation of interventional and surgical therapies. In this article, the past, present, and future prospect of this promising imaging modality will be presented.

\section{Developmental History of Percutaneous Cardioscopy}

Intracardiac observation using a rigid cardioscope in animals was performed by Allen et al. in 1922 [1] and by Harken et al. in 1943 [2]. In 1956, Sakakibara et al. [3] employed a rigid cardioscope to observe a septal defect during open-heart surgery. They also observed aortic valves using the same cardioscope in 1958 [4].

Difficulties in producing a thin endoscope that can safely be introduced percutaneously into the cardiac chambers, and equipment that can displace blood, meant that about 29 years elapsed before Uchida and his coworkers successfully performed percutaneous cardioscopy in patients $[5,6]$. Although this new modality of diagnosis is now performed routinely in a few selected institutions, it has yet to be adopted on a global scale.

In 1975, a 9-F fiberscope was developed in collaboration with Olympus Corporation, Tokyo. This cardioscope was introduced through an 11-F hard-tipped guiding catheter into a canine left ventricle, but was abandoned due to marked damage to the endocardial surface. In 1976, a 10-F balloon-tipped guiding catheter was developed. This catheter allowed the passage of a 6-F fiberscope. However, this cardioscope also had to be abandoned because the balloon became frosty during use due to the temperature difference between the saline used for balloon dilatation and the blood in the ventricle. In the same year, a fiberscope was devised 
with a balloon at its tip. This fiberscope had a central lumen through which warmed saline at body temperature could be infused to dilate the balloon. The balloon was pushed against the endocardial surface to observe changes through the dilated balloon. However, introduction of this fiberscope into the left ventricle was very difficult because a guide wire could not be used, and if used in combination with a guiding catheter, a big catheter had to be used to allow the fiberscope to pass through. This fiberscope was not used clinically. In 1983, a 9-F balloon-guiding catheter was devised in collaboration with Clinical Supply Company, Gifu, Japan. When inflated with $\mathrm{CO}_{2}$, the balloon protruded more distally than the shaft tip to form a dead space between the target and the balloon, at the same time preventing damage to the myocardium by the shaft tip. In combination with a 5-F fiberscope, this balloon catheter enabled percutaneous transluminal observation of the cardiac chambers and valves (Fig. 1). This cardioscopy system is now routinely used clinically for observation, not only of the cardiac chambers and valves, but also of the great vessels including the pulmonary arteries, caval veins, and aorta.

\section{Cardioscopy System}

A cardioscopy system comprises a light source, 4.5-F fiberscope, 9-F guiding balloon catheter, intensified chilled coupled device (ICCD) camera, camera controller, DVD recorder, and television monitor.

The fiberscope (AF 14, Olympus Corporation) is a 4.5-F fiberscope containing 3,000 glass fibers for image guidance and 300 glass fibers for light guidance. The fiberscope is passed through a 9-F guiding balloon catheter (Clinical Supply Company). The balloon is inflated with $\mathrm{CO}_{2}$. The catheter has a $\mathrm{Y}$ connecter at the proximal end: one channel for fiberscope insertion and another for saline flushing. White balance of the cardioscope is adjusted using white gauze that is immersed in saline solution as the white color (Fig. 1) [7-10].

\section{Cardioscopy Procedures}

\section{Left Ventricle}

Usually, following coronary angiography and left ventriculography, a guiding balloon catheter is introduced into the left ventricle and the balloon is inflated with $\mathrm{CO}_{2}$. Next, a fiberscope is introduced via the guiding catheter to place the fiberscope tip at the distal most end of the guiding catheter. The balloon is then gently placed against the endocardial surface. Since the balloon protrudes $5 \mathrm{~mm}$ ahead of the catheter tip, the distance between the fiberscope tip and the endocardial luminal surface is

Fig. 1 Cardioscope and cardioscopy procedure. A, Cardioscope. $\mathrm{a}=$ shaft of guiding balloon catheter. $\mathrm{b}=$ balloon. $\mathrm{c}=$ fiberscope. $\mathbf{B}$, Observation of the left ventricle (LV). $\mathrm{a}=$ guiding balloon catheter in this and in $\mathbf{c}$ to $\mathbf{e}$. $\mathrm{c}=$ fiberscope in this and in $\mathbf{c}$ to e. $\mathbf{C}$, Observation of tricuspid valve (TV). $d=$ guidewire in this and in $\mathbf{d}$ and e. D, Observation of aortic valve (AoV). E, Observation of mitral valve (MV). LA, left atrium; $\mathrm{RA}$, right atrium; $\mathrm{RV}$, right ventricle

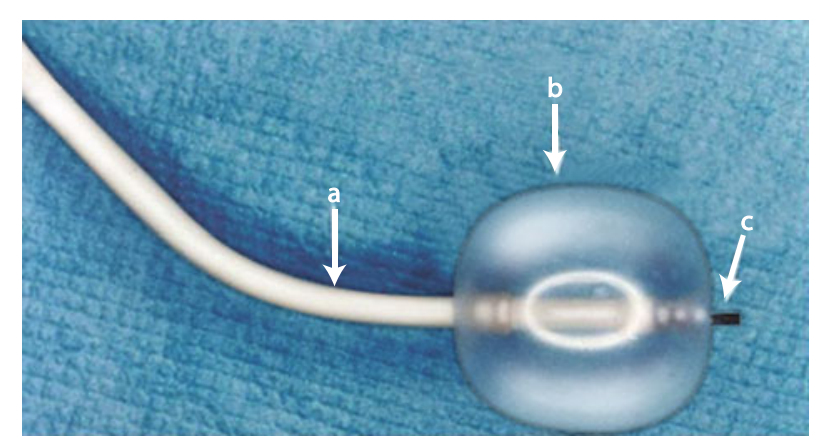

A

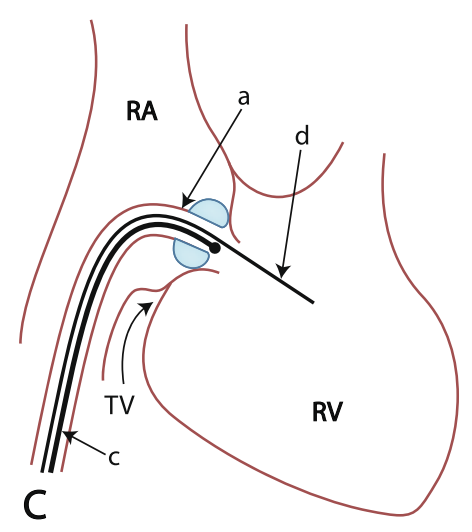

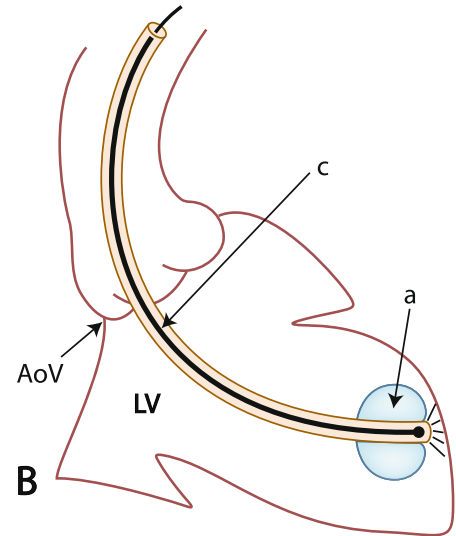
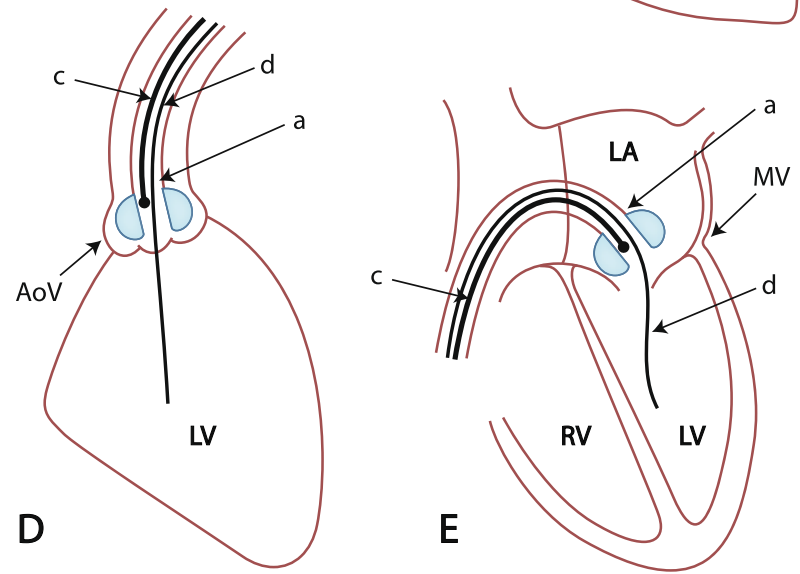
maintained at almost $5 \mathrm{~mm}$ [11]. The diameter of the visual field is approximately $1.2 \mathrm{~cm}$ in saline. Heparinized saline solution $(10 \mathrm{IU} / \mathrm{mL})$ is then infused at a rate of $10 \mathrm{~mL} / \mathrm{s}$ using a power injector for $5 \mathrm{~s}$ to displace the blood between the endocardial surface and the fiberscope. The guiding balloon catheter is pre-shaped to easily locate on the targeted wall segment; an " $\mathrm{S}$ " or "crank" configuration for the anterior, apical, and inferior wall segments; a "J" configuration for lateral wall segment; and an "L" configuration for the high posterior wall segment (Fig. 1B) [10].

\section{Right Ventricle}

Observation of the right ventricle is essentially the same as for the left ventricle. Use of a guiding balloon catheter "J" or " $U$ " configuration is recommended.

\section{Right Atrium}

The free wall of the right atrium and the atrial septum can be observed using a " $\mathrm{J}$ " configuration guiding balloon catheter.

\section{Left Atrium}

The guiding balloon catheter must be introduced transseptally from the right atrium into the left atrium for observation of the left atrial wall.

\section{Cardiac Valves}

For observation of the aortic, mitral, and tricuspid and pulmonary valves, it is necessary to use a guidewire to anchor the catheter tip for observation, as shown schematically in Fig. $1 \mathrm{C}$ to E $[7,10]$.

\section{Measurement of Lesion Sizes}

Measurement of lesion sizes is beyond the scope of present cardioscopy systems because it uses a fish-eye lens. Nevertheless, lesion sizes can be roughly assessed using the diameter of a guidewire tip that is placed on or adjacent to the lesion.

\section{Cardioscope-Guided Endomyocardial Biopsy System}

A cardioscope-guided biopsy system has also been devised to safely guide endomyocardial biopsy and to confirm changes in the biopsied portion. A 1.6-F fiberscope is attached to a bioptome. This system is introduced through either a 9-F guiding balloon catheter or a 9-F soft-tipped catheter into a cardiac chamber, and a biopsy is taken while observing the area to be biopsied [7, 12].

\section{Combination of Cardioscopy and Intracardiac Ultrasound}

The cardiac chambers and valves are usually observed by cardioscopy in combination with intracardiac ultrasound (ICUS). An ICUS probe is introduced through the right femoral artery into the left ventricle, guided by a 0.035 -inch guide wire. The guide wire is advanced to the apex first, and then the probe is advanced to the apex. Use of a radiofocus guide wire (Terumo Company, Tokyo) is recommended because it is very steerable. Pulling back the probe slowly, pineapple-like slices of the left ventricle from the apex to the aortic valve can be successively obtained. A $15-\mathrm{MHz}$ probe is usually used for a normalsized left ventricle. When the ventricle is large, a $12-\mathrm{MHz}$ probe is used to cover the entire chamber.

For right heart examinations, an ICUS probe is introduced through the right femoral vein into the pulmonary artery, guided by a 0.035 -inch guide wire. The probe is slowly pulled back toward the right atrium. By this maneuver, pineapple-like slices of the right ventricle can be successively obtained. Details of this procedure are described elsewhere $[10,13]$.

\section{Observation of the Cardiac Chambers}

\section{Coronary Artery Disease}

Endocardial color has been reported to indicate the severity of myocardial ischemia and fibrosis. The endocardial surface is brown in patients without heart disease when observed by flushing saline solution. The endocardial color of the left ventricular wall in patients with coronary artery disease is classified as brown, light brown, pale (bluish white closely resembling the endocardial color of the Langendorff heart in which the blood is replaced by an artificial solution [14]), and white (Fig. 2). Regional left ventricular contraction assessed using ICUS is usually normokinetic, normokinetic, hypo-toakinetic, and akinetic-to-dyskinetic, respectively (Fig. 2). These endocardial colors, however, do not correlate significantly with the severity of stenosis of the irrigating epicardial artery or collateral development, suggesting that the regional microcirculation is the determinant of regional blood flow and accordingly endocardial color [7]. White trabecular edges are characteristic of vasospastic angina. Transient but severe ischemia due to coronary spasm may cause fibrosis of the trabecular edges that are most susceptible to ischemia [7]. 

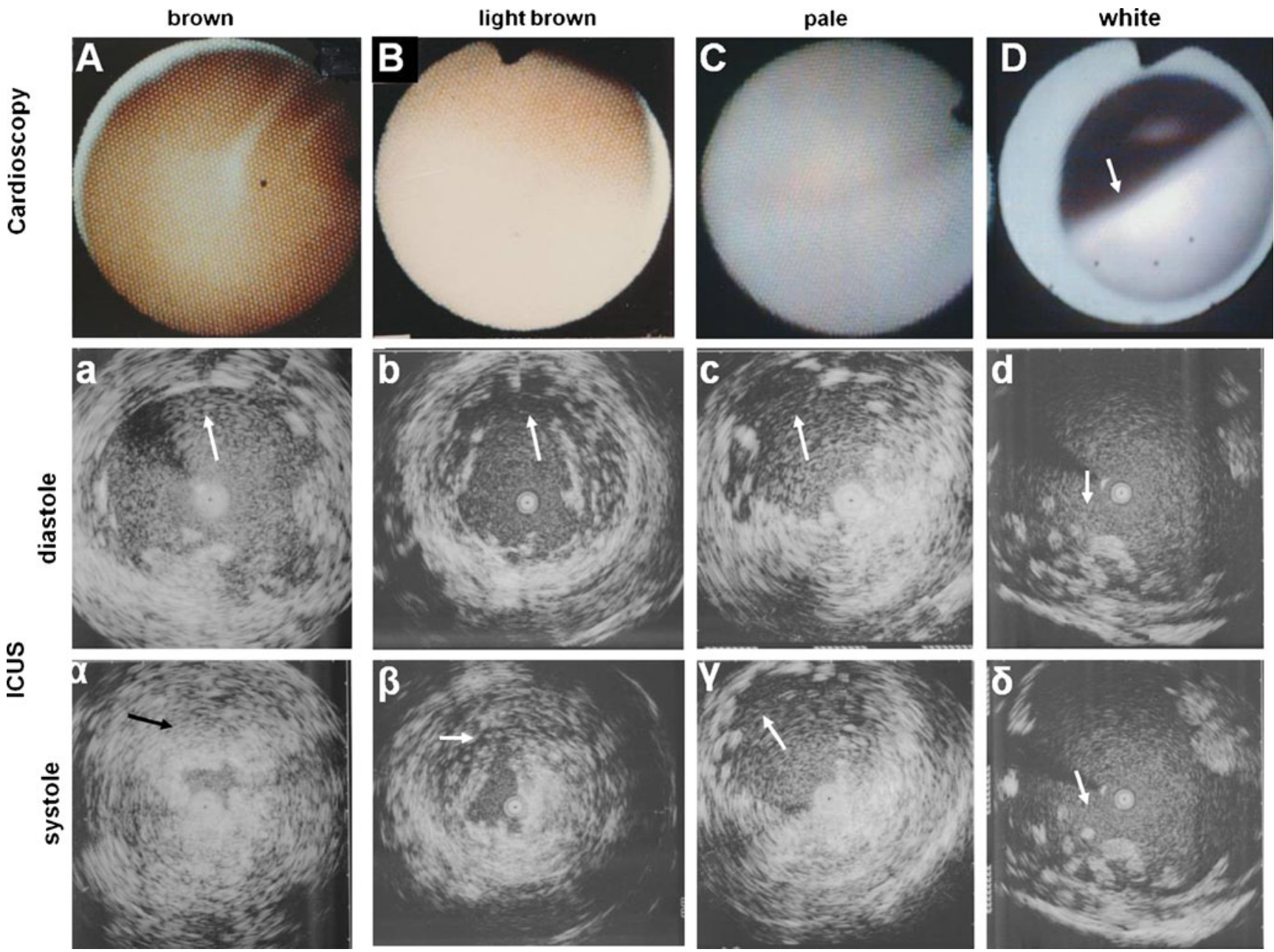

Fig. 2 Left ventricular endocardial color in patients with coronary artery disease. From $\mathbf{A}$ to $\mathbf{D}$, Brown, light brown, pale, and white. Arrow in $\mathbf{d}=$ atrophic trabeculae. a to d, Corresponding intracardiac ultrasound images at diastole. $\alpha$ to $\delta$, Corresponding intracardiac ultrasound images at systole. From $\alpha$ to $\delta$, Normokinetic, normokinetic, hypokinetic anterior wall (arrow), and akinetic inferior wall (arrow), respectively. Arrows in a to $\mathbf{c}$ and $\alpha$ to $\gamma$, anterior wall. Arrows in $\mathbf{d}$ and $\boldsymbol{\delta}$, inferior wall

\section{Myocarditis}

Endomyocardial biopsy is essential to make a definite diagnosis of myocarditis. However, when fluoroscopy is used to guide endomyocardial biopsy, chance decides whether or not the diseased myocardium is biopsied, possibly leading to misdiagnosis. If endocardial surface changes are indicative of the stage of myocarditis, staging of myocarditis can be performed by percutaneous cardioscopy, enabling observation of the cardiac chambers from within, and targeted cardioscope-guided biopsy can be performed for accurate histological diagnosis.

Cardioscope-guided endomyocardial biopsy was performed in 59 patients with idiopathic myocarditis. Left ventricular endocardial color and histological changes were compared. Cardioscopic follow-up studies were performed for 6 months. In contrast to the brown color seen in patients with normal histology, the endocardial surface was red, milky white, purple, white, or yellowish brown in patients with idiopathic myocarditis. Biopsy specimens obtained from red and milky white segments exhibited histological changes indicative of acute myocarditis, purple segments of active chronic myocarditis, and yellowish brown and white segments of inactive chronic myocarditis (Fig. 3) [13, 15, 16].

Cardioscopic follow-up studies revealed that red and milky white surface was changed to purple or white, and purple surface was often changed to white or yellowish brown, indicating transformation of inflammatory stages. Thus, left ventricular endocardial colors that were observed by cardioscopy represented histological stages of idiopathic myocarditis. This imaging tool is therefore considered to be feasible for staging and follow-up of idiopathic myocarditis $[13,15,16]$. 
Fig. 3 Left ventricular endocardial color in patients with idiopathic myocarditis. A, Normal control. Arrow indicates trabeculae. $\mathbf{B}$ to $\mathbf{F}$, Red indicating acute myocarditis, milky white indicating acute myocarditis, purple indicating active chronic myocarditis, white indicating inactive chronic myocarditis, and yellowish brown indicating inactive chronic myocarditis as determined by biopsy, respectively

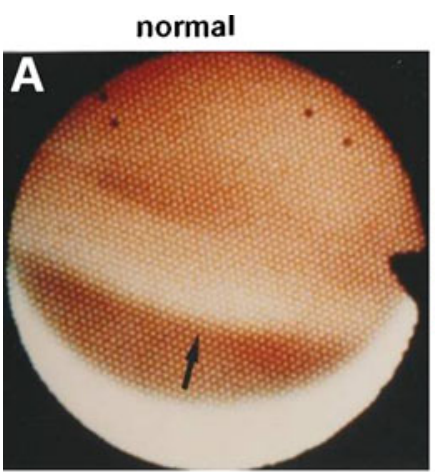

purple

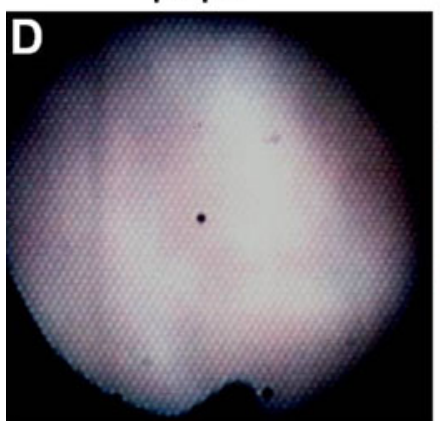

red

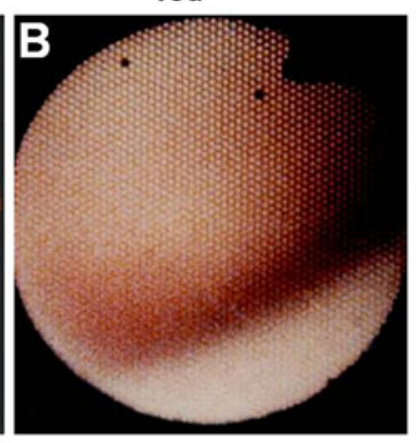

white

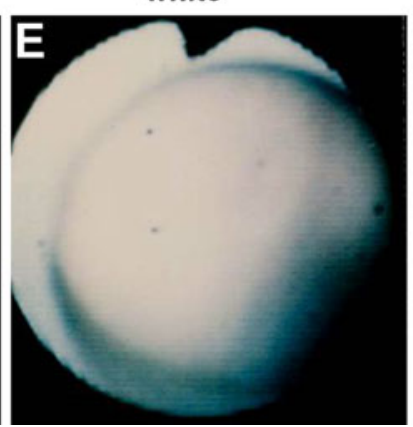

milky white

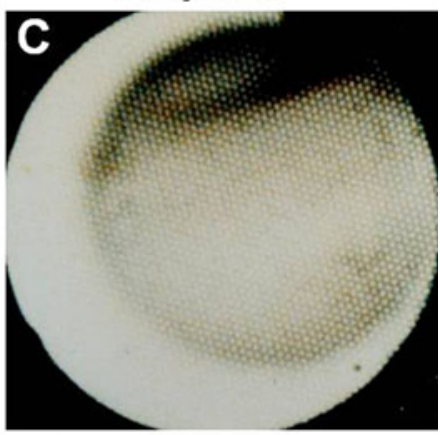

yellowish brown

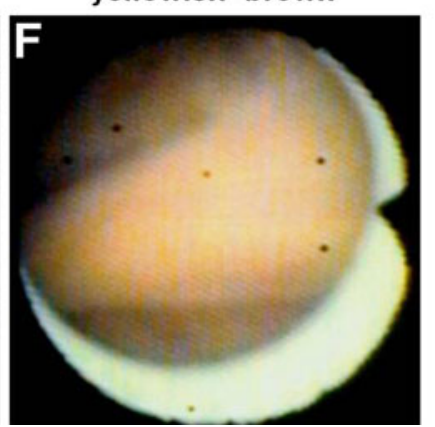

\section{Idiopathic Dilated Cardiomyopathy}

In general, the left ventricular endocardial color is white or yellowish brown and trabeculae are thin and atrophic in patients with dilated cardiomyopathy. Since the endocardial color resembles that of inactive idiopathic myocarditis, histological examination is essential to make a definitive diagnosis [17].

\section{Idiopathic Hypertrophic Cardiomyopathy}

The most outstanding change in the left ventricle in patients with hypertrophic cardiomyopathy is the presence of thick trabeculae. These are usually light brown at first, later turning white due to fibrosis in the dilated phase [18].

\section{Subendocardial Microvessels}

The coronary microvessels play a direct and critical role in determining the extent and severity of myocardial ischemia and symptoms, and preservation of cardiac function. Participation of microvessel dysfunction is suspected, but not confirmed, in the slow-flow or no-flow phenomenon associated with percutaneous coronary intervention [19], Takotsubo cardiomyopathy [20], peripartum cardiomyopathy [21], syndrome X [22], and microvessel angina [23].

Direct visualization may help to elucidate the mechanisms of these heart diseases. However, there are no clinically available methods for direct imaging of coronary microvessels in vivo.
The subendocardial microvessels were observed using cardioscopy in patients with coronary artery disease. It was revealed that subendocardial arterial and venous microvessels located in normokinetic-to-hypokinetic left ventricular wall segments were filled with the blood during diastole and collapsed during systole. In contrast, subendocardial arterial and venous microvessels located in akinetic-to-dyskinetic wall segments were filled with the blood during systole and collapsed during diastole. No significant correlation was found between these changes and the severity of stenosis of the irrigating epicardial coronary arteries or collateral development. These findings suggest that the contractile state of the myocardium is the main determinant of the timing of perfusion of the subendocardial microvessels in patients with coronary artery disease [24].

\section{Left Ventricular Thrombus}

It is well known that a fibrillating left atrium is a site of thrombus formation, acting as a major supply source of thromboemboli in cerebral ischemic attacks [25-28]. It is also known that the left ventricle is a site of thrombus formation in the post-infarction state [29]. Other heart diseases such as peripartal cardiomyopathy [30], idiopathic dilated cardiomyopathy [31], acute myocarditis [32], and antiphospholipid syndrome [33] may cause cerebral embolism [34, 35]. However, the exact incidence of left ventricular thrombus (LVT) in various categories of heart disease is unclear due to the lack of systematic surveys. 
In a previous cardioscopy study, LVT was detected in $26 \%$ of 258 patients with heart diseases. Cardioscopically, LVT are classified by shape as globular (protruding) and mural (lined), and by color as red, white, and yellow (Fig. 4). The majority of LVTs detected were mural. LVT was detected in $12.5 \%$ of patients with stable angina, $0 \%$ with unstable angina, $45.2 \%$ with acute myocardial infarction, $23.5 \%$ with old myocardial infarction, $61.9 \%$ with idiopathic acute myocarditis, $44.3 \%$ with idiopathic chronic myocarditis, $33.3 \%$ with rheumatic valvular disease, $25.7 \%$ with idiopathic dilated cardiomyopathy, and $8.0 \%$ with idiopathic hypertrophic cardiomyopathy. The LVT detection rates using cardioscopy, left ventriculography, noncontrast echocardiography, and contrast echocardiography were $30.2 \%, 2.7 \%, 1.9 \%$, and $7.0 \%$, respectively $[36,37]$. Thus, LVT is common in patients with various heart diseases, especially acute myocardial infarction and acute myocarditis, and although invasive, cardioscopy is more sensitive in detecting LVT than left ventriculography or non-contrast and contrast echocardiography.

\section{Observation of Cardiac Valves}

Direct observation of the cardiac valves in the beating heart was previously beyond the scope of any available imaging modalities. Figure 5 shows the morphology and motion of
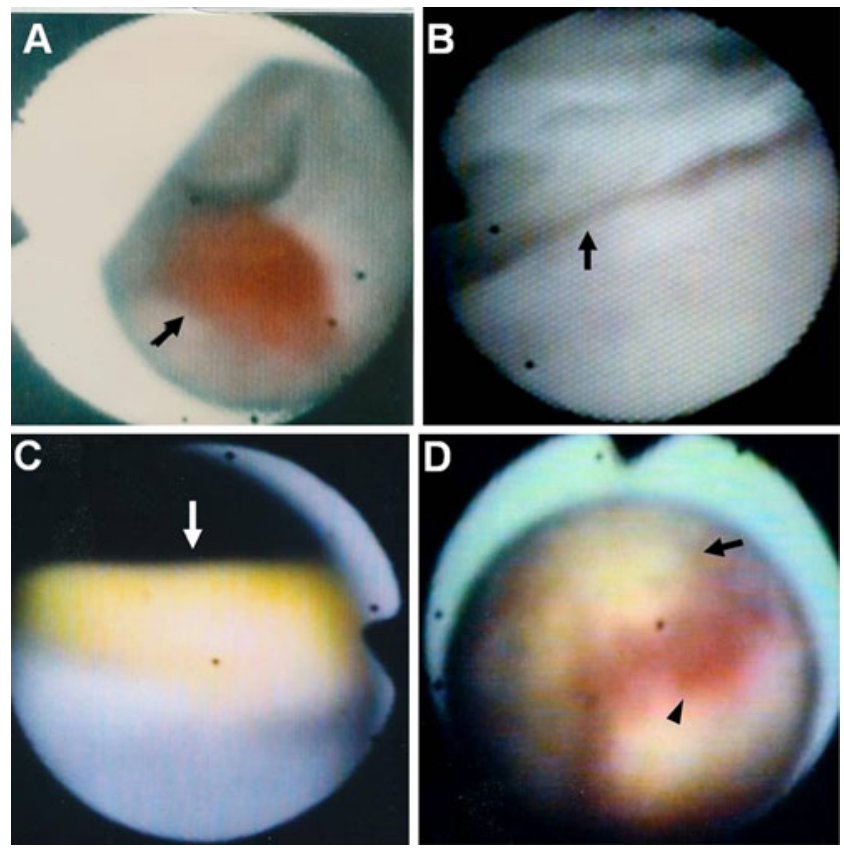

Fig. 4 Color of left ventricular thrombi (LVT). A, Red LVT (arrow) in a 66-year-old man with old myocardial infarction (OMI). B, White LVT (arrow) in a 47-year-old woman with chronic myocarditis. C, Yellow LVT (arrow) in a 50-year-old man with OMI. D, Red-andyellow in a mosaic pattern LVT in a 71-year-old man with OMI. Arrow indicates yellow LVT. Arrowhead indicates red LVT normal aortic and mitral valves. Figure 6 shows the surface morphology of diseased aortic cusp and mitral leaflets as visualized using cardioscopy [38, 39].

Cardioscopy and intracardiac ultrasound allow us to observe not only morphological changes, but also to examine precisely the motion of the cardiac valves.

\section{Evaluation of Medical, Interventional, and Surgical Therapies}

\section{Effects of Nitroglycerine}

The subendocardial myocardial layer is most susceptible to ischemia. To improve blood flow in this layer is therefore an essential requisite for the treatment of coronary artery disease. Nitroglycerine (NTG) is a well-known antianginal agent. The question of whether NTG increases blood flow to the subendocardial myocardial layer is controversial, however.

Changes in subendocardial myocardial blood flow (SMBF) induced by the intravenous administration of $200 \mu \mathrm{g}$ of NTG were examined using cardioscopy in patients with coronary artery disease.

Figure 7 shows an example of NTG-induced recovery of myocardial blood flow in a patient with angina pectoris. On administration of NTG, the endocardial color changed to red in brown and light brown segments, indicating arterial blood filling. Variable changes were seen in pale segments: turned purple, indicating venous blood filling; turned red, indicating arterial blood filling, or showed no change. No changes were seen in the white segments, indicating no blood filling. NTG-induced changes in endocardial color were therefore closely related to the control color. Although there was a tendency for arterial blood filling to occur in segments irrigated by a less stenotic artery, with developed collaterals and with well preserved contraction, the difference was not statistically significant [40, 41]. It is conceivable that regional microvessels, and not large epicardial coronary arteries, directly mediate the effects of NTG on SMBF.

\section{Evaluation of Percutaneous Coronary Interventions}

The effects of percutaneous coronary interventions were examined using cardioscopy in patients with acute coronary syndrome. Pale endocardial color often turned dark red indicating reperfusion hyperemia.

\section{Evaluation of Percutaneous Transseptal Mitral} Commissurotomy

Figure 8 shows the posterior commissure of the mitral valve in a patient with rheumatic mitral stenosis. The fused commissure was separated using percutaneous transseptal 

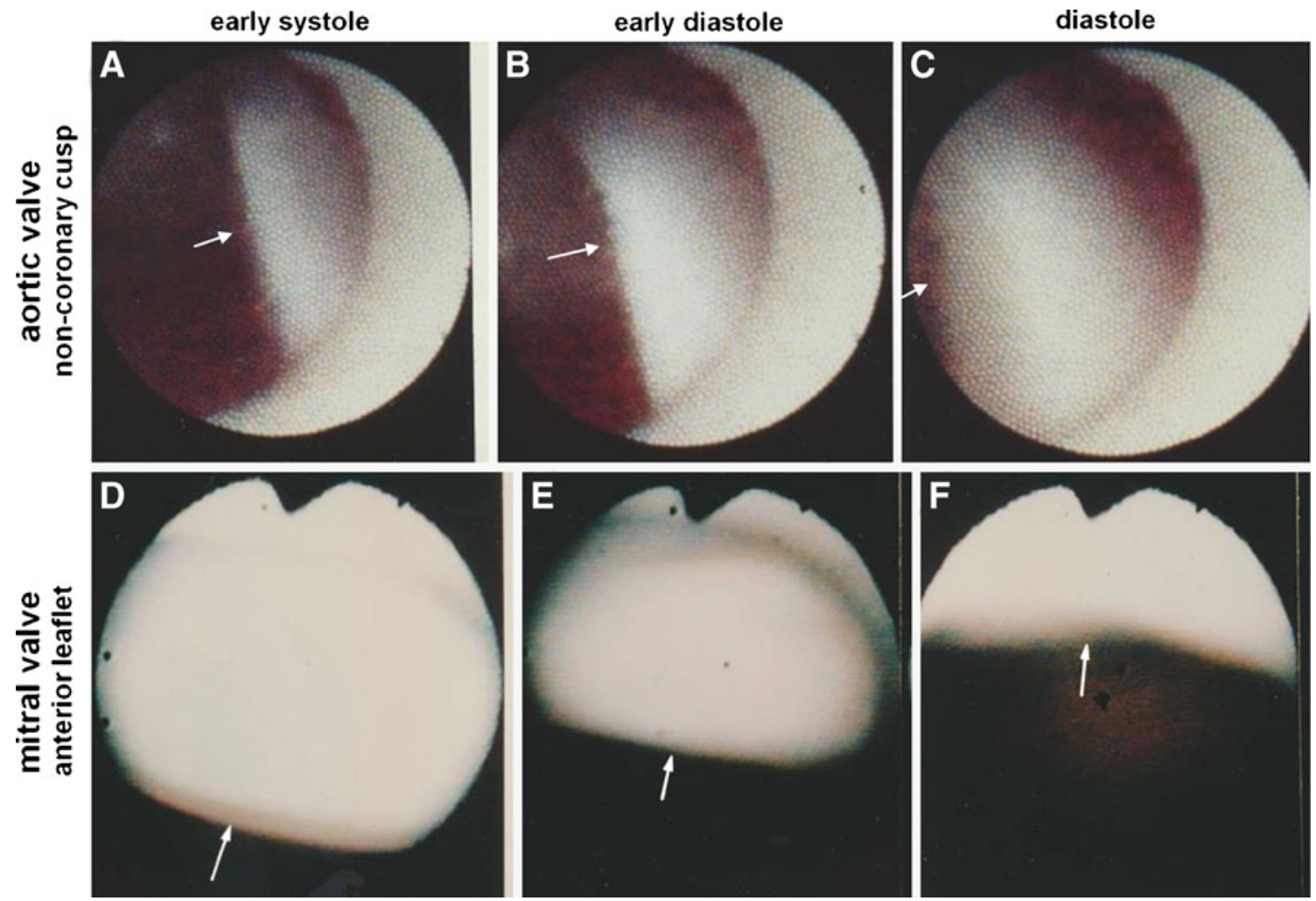

Fig. 5 Cardioscopic images of normal aortic and mitral valves. A to C, Process of aortic valve closing. Arrows indicate noncoronary cusp. D to F, Process of mitral valve opening of normal mitral valve. Arrows indicate anterior leaflet
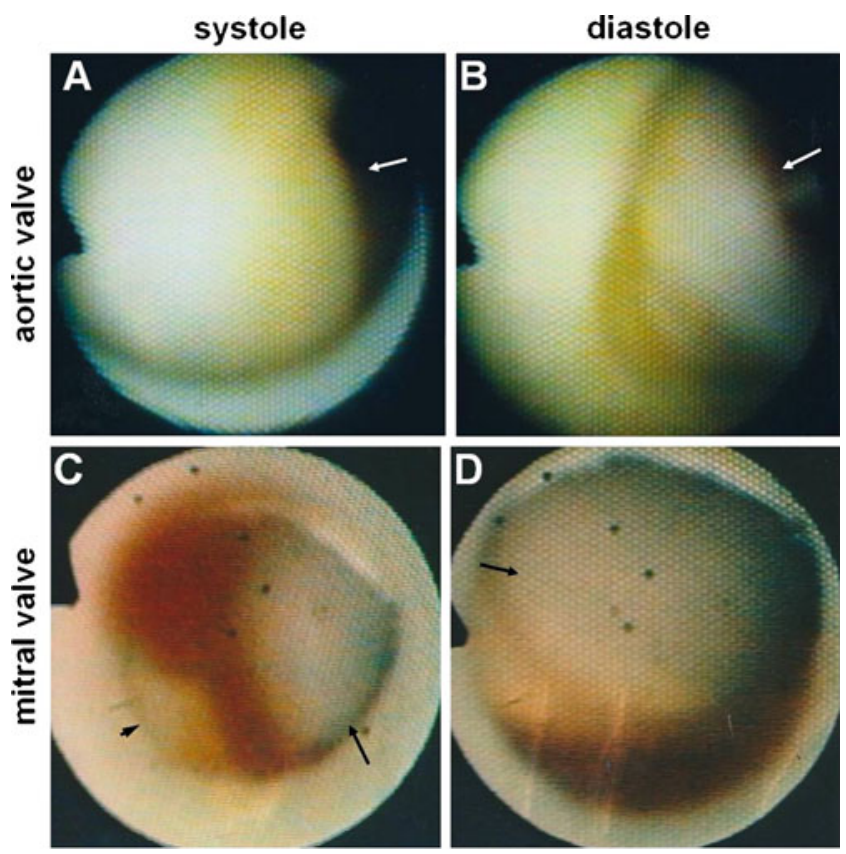

Fig. 6 Diseased aortic and mitral valves. $\mathbf{A}$ and $\mathbf{B}$, Yellow and thick noncoronary cusp in a patient with rheumatic aortic regurgitation during systole and diastole, respectively (arrows). C and D, Yellow and thick posterior mitral leaflet (arrowhead) and anterior leaflet (arrows) during systole and diastole in a patient with rheumatic mitral steno-regurgitation, respectively. (From Uchida [10]; with permission) mitral commissurotomy. Cardioscopy can be used for the evaluation of catheter-based commissurotomy not only of the mitral valve, but also other cardiac valves.

\section{Evaluation of Cardiac Surgery}

Cardioscopy can be used for evaluation of surgery of congenital heart diseases such as atrial septal defect, anomalous pulmonary vein drainage, and aortic disease.
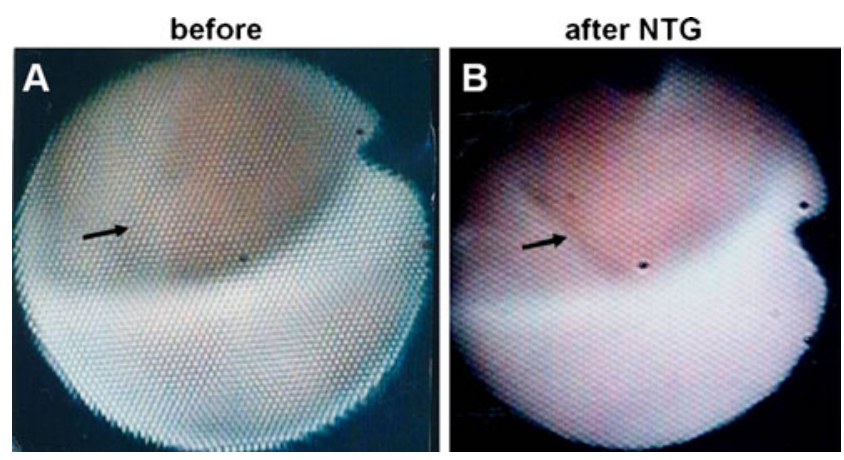

Fig. 7 Effects of nitroglycerin (NTG) on subendocardial myocardial blood flow. A and B, Before and 3 min after the intravenous injection of $200 \mu \mathrm{g} \mathrm{NTG}$, respectively. The endocardial color changed from white to red, indicating increased blood flow (arrow in B). (From Uchida [7]; with permission) 

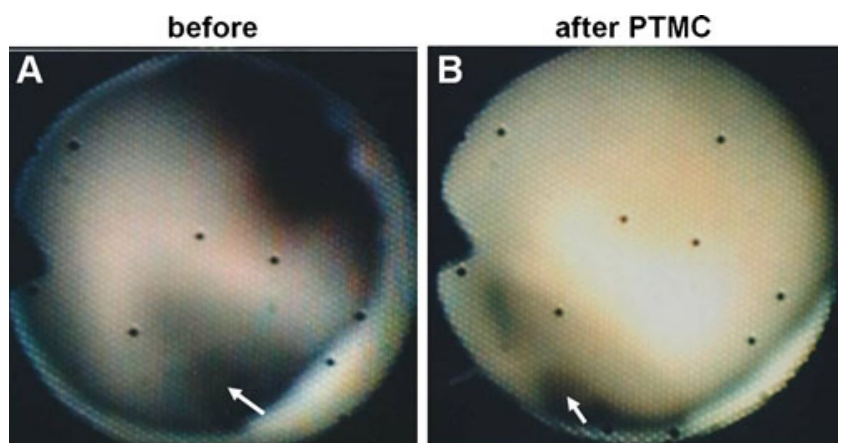

Fig. 8 Evaluation of percutaneous transseptal mitral commissurotomy (PTMC) in a 44-year-old woman with rheumatic mitral stenosis. A Fusion of the posterior commissure (arrow). B, Separation of the fused commissure by PTMC (arrow). (From Uchida [10]; with permission)

Observation of artificial valves should be avoided because of the risk of valvular damage [10].

\section{New Cardioscopic Modalities}

\section{Dye-Staining Cardioscopy}

Dye-staining cardioscopy, using a dye as an indicator of blood flow, is useful for the identification of regional myocardial
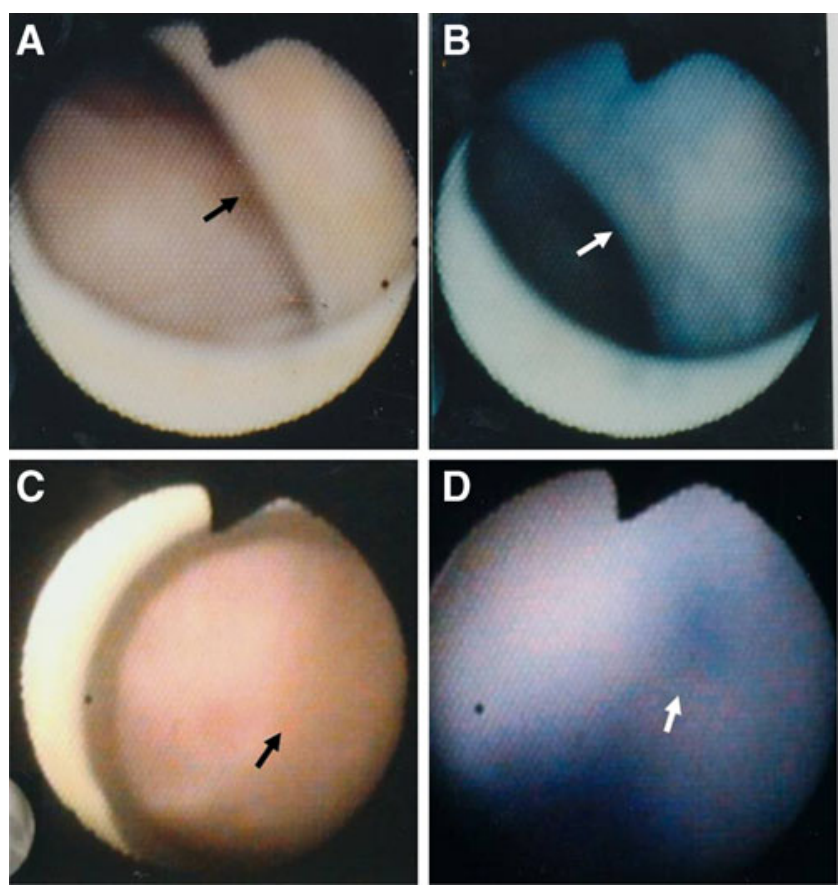

Fig. 9 Dye-staining cardioscopy. A and B, Left ventricular endocardial color before and after intracoronary injection of Evans blue dye in a subject without coronary artery disease. Note diffuse staining, indicating normal blood flow (arrow in B). C and D, Left ventricular endocardial color before and after intracoronary injection of Evans blue dye in a patient with old myocardial infarction. Note patchy staining (arrow in D). (From Uchida et al. [42•]; with permission) blood flow. During observation of a wall segment, a selective bolus injection of $1 \mathrm{~mL}$ of $2 \%$ Evans blue solution into the irrigating coronary artery results in staining of the wall segment when the artery is patent, but no or partial staining when the artery is obstructed or stenosed. Figure 9B shows diffuse staining of the myocardium following selective intracoronary injection of Evans blue dye in a patient with chest pain syndrome, indicating normal preservation of myocardial blood flow. Figure 9D shows patchy staining of the myocardium in a patient with old myocardial infarction, indicating regional disturbance of myocardial blood flow.

Dye-staining cardioscopy was performed before and after coronary stent implantation in patients with acute myocardial infarction. This showed that despite successful recanalization of the obstructed epicardial coronary artery, the endocardial surface was not necessarily stained with Evans blue, indicating that coronary microcirculation was not necessarily restored by apparently successful epicardial coronary recanalization $[42 \cdot]$.

\section{Fluorescence Cardioscopy}

Fluorescein generates fluorescence at $520 \mathrm{~nm}$ when excited by $470 \mathrm{~nm}$ light, and is routinely used for detection of
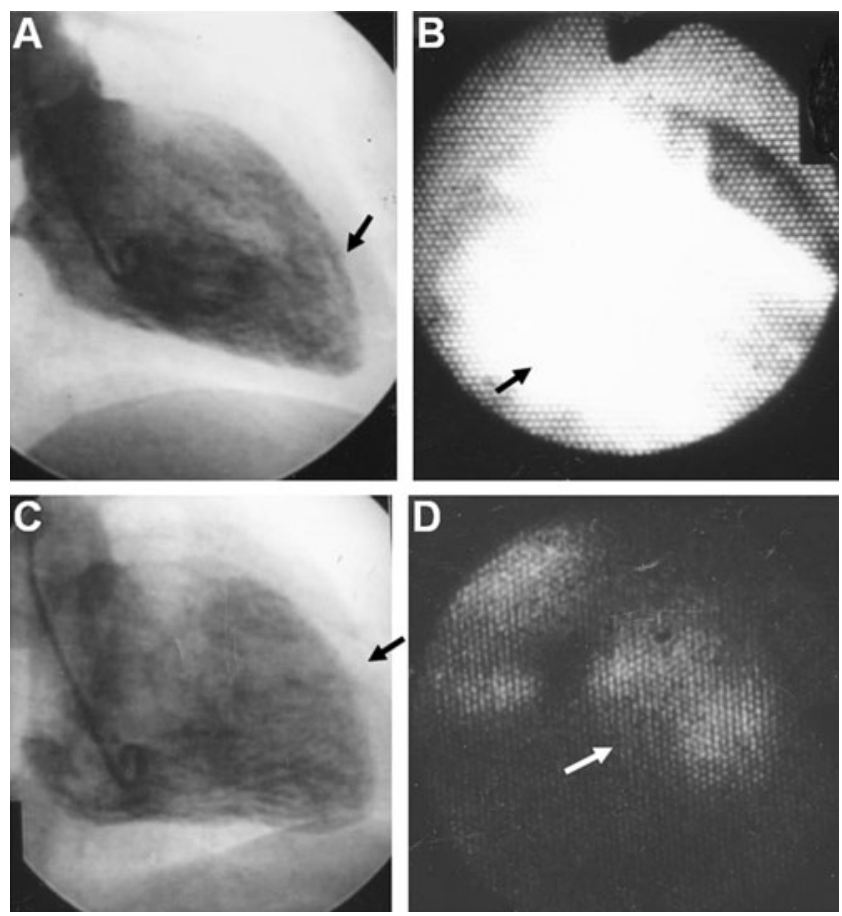

Fig. 10 Fluorescence cardioscopy. A, Left ventriculograms in a patient with chest pain syndrome. The apical segment shows diffuse fluorescence after intravenous injection of fluorescein $\mathbf{B}$, indicating normal myocardial tissue fluid flow. $\mathbf{C}$, Left ventriculograms in a patient with old myocardial infarction. The apical segment shows patchy fluorescence, indicating regional disturbance of fluid flow $\mathbf{D}$. (From Uchida et al. [43••]; with permission) 


\section{fluorescence cardioscopy}

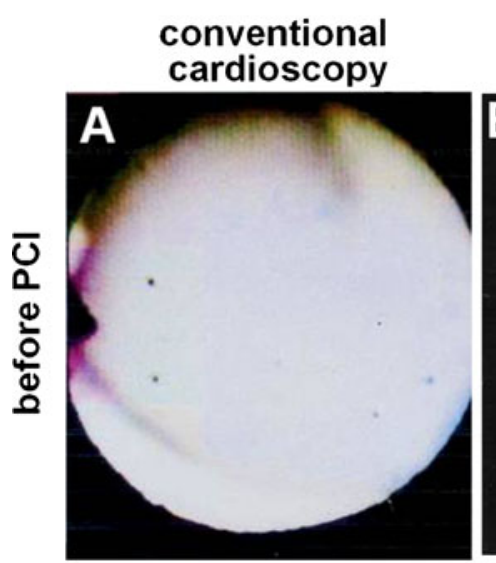

1
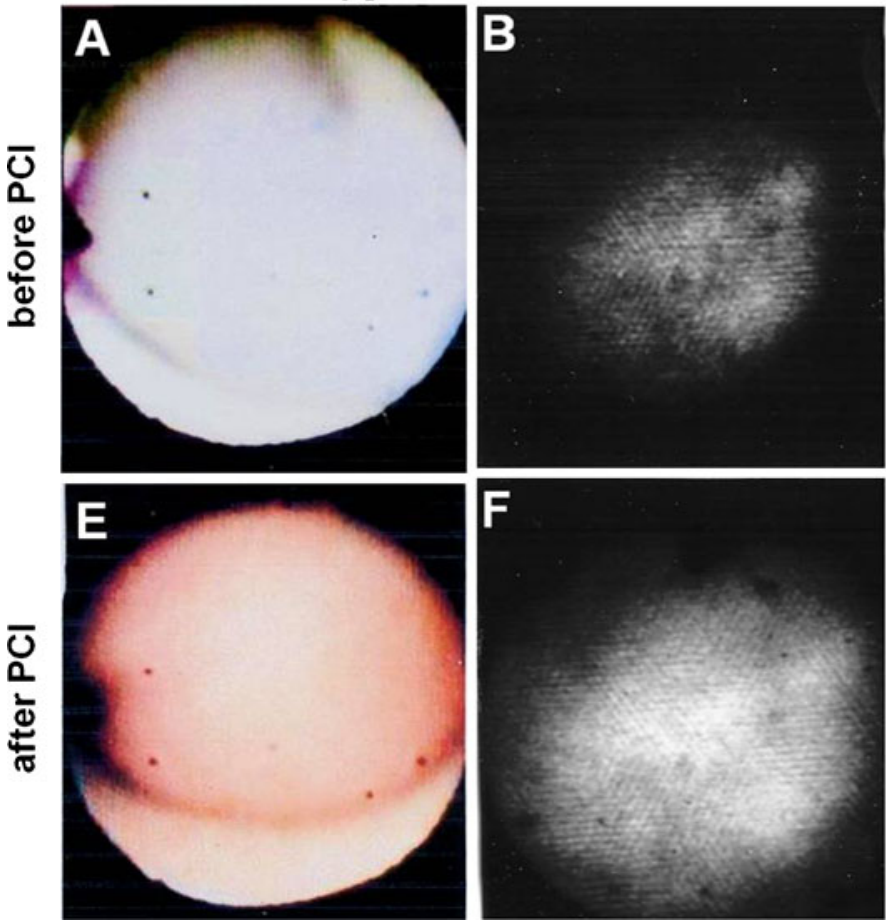

3
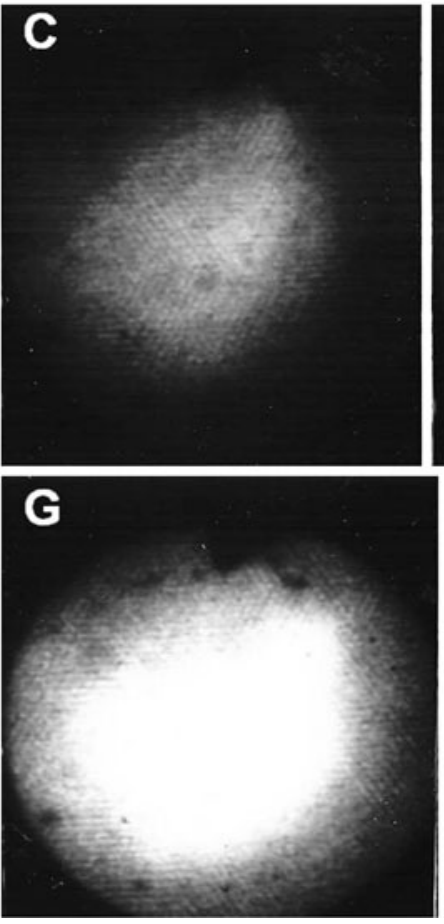

$6 \mathrm{~min}$
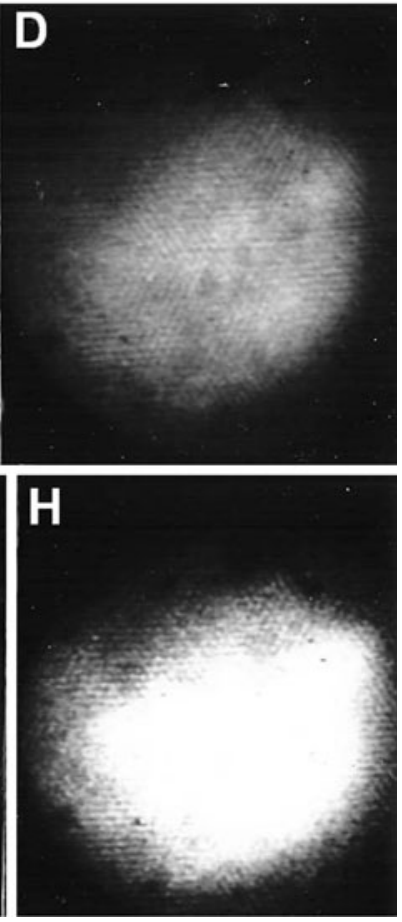

Fig. 11 Fluorescence cardioscopy before and after percutaneous coronary intervention (PCI). A and $\mathbf{E}$, Conventional cardioscopy before and after PCI, respectively. The endocardial color changed from white to red, indicating restoration of blood flow. B to D, Time-

retinal artery microaneurysms in patients with diabetes mellitus. When injected into a vessel, its fluorescence is masked by blood cells, but after diffusion through the vascular wall into the tissues, it exhibits fluorescence. Therefore, the presence of fluorescence in a tissue indicates existence of tissue fluid flow and accordingly blood flow in the irrigating vessel.

Figure 10B shows diffuse staining of the left ventricular endocardial surface by the intravenous injection of fluorescein in a patient with chest pain syndrome, indicating preserved myocardial tissue fluid flow. Figure 10D shows patchy staining of the myocardium in a patient with old myocardial infarction, indicating tissue flow disturbance and accordingly regional blood flow disturbance $[43 \cdot \bullet]$.

After successful percutaneous coronary interventions, fluorescence appears or its intensity is increased, indicating restoration of tissue fluid flow (Fig. 11). However, myocardial tissue flow does not necessarily normalize, indicating that successful epicardial coronary recanalization does not necessarily result in recovery of coronary microcirculation.

These results obtained using dye-staining and fluorescence cardioscopy suggest a need for new modalities for treatment of coronary microcirculatory disturbances. course changes in fluorescence intensity before PCI. F to $\mathbf{H}$, Timecourse changes in fluorescence intensity after PCI. An increase in fluorescence intensity indicates flow restoration is evident. (From Uchida et al. [43••]; with permission)

\section{Future Directions for Cardioscopy}

\section{Cardioscope-Guided Intracardiac Surgery}

Cardioscope-guided myotomy, myectomy, and valvulotomy have been trialed in animals in anticipation of their clinical application [44-47]. These therapeutic modalities will soon be available for application in clinical situations.

\section{Cardioscope-Guided Trans-endocardial Angiogenic} and Myogenic Therapy

About $15 \%$ of patients with ischemic heart disease are not indicated for surgical or other interventions. At present, no other curative treatments are available for them.

Myocardial salvage through the formation of new blood vessels by either angiogenesis or vasculogenesis (angiogenic therapy) is one promising therapeutic modality for these patients. Cardioscope-guided trans-endocardial angiogenic therapy has been trialed in animals [48]. Using dyestaining and fluorescence cardioscopy, pin-point angiogenic and myogenic therapy can be performed with more precision in the clinical situation. 


\section{Conclusions}

Recent advances in cardioscopy technology enable us to observe the interior of the heart. This imaging technology is now used for the diagnosis of myocardial and valvular diseases, evaluation of the severity of myocardial ischemia, interventional and surgical treatments, and for guidance of endomyocardial biopsy. This technique will be employed for the guidance of transcatheter interventional and surgical treatments of various heart diseases in the near future.

Disclosure No potential conflict of interest relevant to this article was reported.

Open Access This article is distributed under the terms of the Creative Commons Attribution Noncommercial License which permits any noncommercial use, distribution, and reproduction in any medium, provided the original author(s) and source are credited.

\section{References}

Papers of particular interest, published recently, have been highlighted as:

- Of importance,

-• Of major importance

1. Allen DS, Graham EA. Intracardiac surgery-a new method. Am Med Ass. 1922;79:1028.

2. Harken DE, Glidden EM. Experiments in intracardiac surgery. II. Intracardiac visualization. J Thorac Surg. 1943;12:566.

3. Sakakibara H, Ichikawa T, Hattori J. An intraoperative method for observation of cardiac septal defect using a cardioscope. Operation. 1956;10:285-90.

4. Sakakibara H, Iijima T, Hattori J, et al. Direct visual operation for aortic stenosis: cardioscope studies. J Int Coll Surg. 1958;29:548-52.

5. Uchida Y, Tomaru T, Nakamura F, et al. Fiberoptic angioscopy of cardiac chambers, valves and great vessels using a guiding balloon catheter in dog. Am Heart J. 1988;118:1297-302.

6. Uchida Y, Ohshima T, Shibuya I. Percutaneous angioscopy of the right side of the heart in humans. Cardiovasc World Rep. 1988;1:13-7.

7. Uchida Y. Clinical application of percutaneous cardioscopy for coronary heart disease. In: Uchida Y, editor. Coronary angioscopy. NY: Futura; 2001. p. 181-233.

8. Uchida Y. Percutaneous cardiovascular angioscopy. In: Abela G, editor. Lasers in cardiovascular medicine and surgery. Boston: Kluwer Academic Press; 1989. p. 399-410.

9. Uchida Y, Fujimori Y, Hirose J. Percutaneous cardioscopy. Jpn Heart J. 1992;33:271-94.

10. Uchida Y. Atlas of cardioangioscopy. Tokyo: Medical View; 1995. p. $94-129$.

11. Uchida Y, Nakamura F, Tsukamoto $T$, et al. Percutaneous ventricular endomyocardial biopsy with angioscopic guidance. Am Heart J. 1989;118:1039-41.

12. Uchida Y, Kanai M, Sakura T. Discrimination of left ventricular myocardial layers by an intracardiac ultrasonography in patients with ischemic heart disease. Jpn Circulat J. 2000;64(Suppl I):172.
13. Hirose J, Sasaki S, Morizuki M, et al. Follow-up study of patients with idiopathic myocarditis by percutaneous cardioscopy. Cardioangioscopy and Laser Cardioangioplasty. 1995;5:33-4.

14. Aylin Arici M, Kilink E, Demir O, et al. Interactions between verapamil an1d digoxin in Langendorff-perfused rat hearts: the role of inhibition of p-glycoprotein in the heart. Basic Clin Pharmacol Toxicol. 2010;20:20-30.

15. Uchida Y, Tomaru T, Nakamura F, et al. Percutaneous cardioscopy of the left ventricle in patients with myocarditis. SPIE. 1992;1642:214-6.

16. Uchida Y, Sakurai T, Kanai M, et al. Relationships between cardioscopic images and histological changes in the left ventricle of patients with idiopathic myocarditis. Eur J Heart Failure. 2011;13:504-12.

17. Uchida Y, Fujimori Y, Hirose J. Percutaneous left ventricular endomyocardial biopsy with angioscopic guidance in patients with dilated cardiomyopathy. Am Heart J. 1990;119:949-52.

18. Fujimori Y, Oshima T, Hirose J, et al. Cardioscopic features of left ventricle in patients with idiopathic hypertrophic cardiomyopathy. Cardioangioscopy and Laser Cardioangioplasty, 1991;4:28.

19. Yilmaz H, Demir I, Uyar Z. Clinical and coronary angiographic characteristics of patients with coronary slow flow. Acta Cardiol. 2008;63:579-84.

20. Danieles DV, Fearon WF. The index of microcirculatory resistance (IMR) in takotsubo cardiomyopathy. Catheter Cardiovasc Interv. 2010;75:77-9.

21. Fizianska A, Walczak E, Glinska Z, et al. Ultrastructural evidence of myocardial remodeling in peripartum cardiomyopathy. Med Sci Monit 2010; 16: CS62-66.

22. Pasqui AL, Pussetti L, Di Renzo M, et al. Structural and functional abnormality of systemic microvessels in cardiac syndrome X. Nutr Metab Cardiovasc Dis. 2005;15:56-64.

23. Mohri M, Koyanagi M, Egarashi K, et al. Angina pectoris caused by coronary microvascular angina. Lancet. 1998;351:1165-9.

24. Uchida Y, Kanai M, Ohsawa $H$, et al. Direct visualization of subendocardial microvessels by percutaneous cardioscopy in patients with heart disease. Circulation. 1998; 98 (Suppl): I-448.

25. Becker RC. Thrombogenesis in atrial fibrillation contributing mechanisms and natural history. $\mathrm{J}$ Thromb Thrombolysis. 2009;27:119-1121.

26. Han SW, Nam HS, Kim SH, et al. Frequency and significance of cardiac sources of embolism in TOAST classification. Cerebrovasc Dis. 2007;24:463-8.

27. Kaviak ES, Kucukoglu H, Yigit Z, et al. Clinical and echocardiographic risk factors for embolization in the presence of left atrial thrombus. Echocardiography. 2007;24:515-21.

28. Bemhardt P, Schmidt H, Hammerstingl C, et al. Atrial thrombi-a prospective follow-up study over 3 years with transesophageal echocardiography and cranial magnetic resonance imaging. Echocardiography. 2006;23:388-94.

29. Siebelink HM, Scholte AJ, Van de Veire NR, et al. Value of contrast echocardiography for left ventricular thrombus detection post-infarcton and impact on antithrombotic therapy. Coron Artery Dis. 2009;20:462-6.

30. Kane A, Mbaye M, Ndiaye MB, et al. Evolution and thromboembolic complications of the idiopathic peripartal cardiomyopathy at Dakar University Hospital: forward-looking study about 33 cases. J Gynécol Obstét Biol Reprod. 2010;14:55-60.

31. Choi SH, Jeong SI, Yang JH, et al. A single-center experience with Intracardiac thrombosis in children with dilated cardiomyopathy. Pediatr Cardiol. 2010;31:264-9.

32. Aboukhoudir F, Rekik S, Hirsch JL. Images in cardiovascular medicine. Live echocardiographic visualization of the migration of a voluminous left ventricular thrombus complicating an acute myocarditis. Circulation. 2009;120:e8-10. 
33. Sen T, Gungor O, Akpinar I, et al. Cardiac involvement in hypereosinophilic syndrome. Tex Heart Inst J. 2009;36:628-9.

34. Buchibinder NA, Yu R, Rosenbloom BE, et al. Left ventricular thrombus and embolic stroke caused by a functional paraganglioma. J Clin Hypertens. 2009;11:734-7.

35. Cianciulli TF, Saccheri MC, Lax JA, et al. Left ventricular thrombus mimicking primary cardiac tumor in a patient with primary antiphospholipid syndrome and recurrent systemic embolism. Cardiol J. 2006;16:560-3.

36. Oshima T, Hirose J, Sasaki M, et al. Detection of mural thrombus of cardiac chambers by percutaneous cardioscopy. Cardioangioscopy and Laser Cardioangioplasty. 1995;5:35-6.

37. Uchida Y, Uchida Y, Sakurai T, et al. Cardioscopic detection of left ventricular thrombi. Proceeding of 24th Annual Meeting of Japanese Association for Cardioangioscopy 2010;10:21.

38. Uchida Y, Ohshima T, Yoshihara F, et al. Percutaneous fiberoptic angioscopy of the cardiac valves. Am Heart J. 1991;121:1791-8.

39. Uchida Y, Percutaneous angioscopy of cardiac chambers and valves. In: Progress in Cardiology, Zipes D, Lea \& Febiger (ed), Boston, Philadelphia, 1991; 163-192.

40. Uchida Y. Effects of nitroglycerin and nicorandil on subendocardial blood flow in patients with ischemic heart disease. Therapeutic Research. 1992;3:93-9.

41. Uchida Y, Uchida Y, Shirai S, et al. Heterogeneous arterial and venous blood filling into the subendocardial myocardium following nitroglycerin administration in patients with coronary artery disease. Proceeding of 24th Annual Meeting of Japanese Association for Cardioangioscopy. 2010; 69.

42. • Uchida Y, Uchida Y, Sakurai T, et al. Imaging of subendocardial myocardial blood flow by dye-staining cardioscopy in patients with coronary artery disease. Int Heart J. 2010; 51: 308-311. Direct visualization of regional subendocardial myocardial blood flow was performed using percutaneous dye-staining cardioscopy in patients.

43. •• Uchida Y, Uchida Y, Koga A, et al. Evaluation of myocardial tissue fluid low by fluorescence cardioscopy in patients with coronary artery disease. Int Heart J 2010; 51: 153-158. Direct visualization of subendocardial tissue fluid flow was performed using percutaneous fluorescence cardioscopy in patients.

44. Uchida Y, Nakamura F, Kido H, et al. Percutaneous cardiomyotomy and valvulotomy with angioscopic guidance. Am Heart J. 1991;121:1221-4.

45. Nakamura F, Miwa F, Uchida Y, et al. Percutaneous intracardiac surgery with cardioscopic guidance. SPIE. 1992;1642:217-20.

46. Nakamura F, Uchida Y, Tomaru $T$, et al. Laser ablation of myocardium with angioscopic guidance. 63th Scientific Sessions of Am Heart Association. Dallas, 1990.

47. Uchida Y, Tomaru T, Nakamura F, et al. Transcatheter treatment of hypertrophic obstructive cardiomyopathy. Jpn Circulat J 1991; (Suppl): 130 .

48. Koga A. Selective arteriogenesis induced by transplantation of bone marrow mononuclear cells with all-trans retinoic acid to ischemic myocardium. J Tokyo Jikei Univ. 2004;119:413-9. 